\title{
La amenorrea como criterio diagnóstico de la anorexia nerviosa*
}

\section{Amenorrhea as a Diagnostic Criterion for Anorexia Nervosa}

Recibido: diciembre 2 de 2011 | Revisado: julio 18 de 2012 | Aceptado: octubre 2 de 2012

\author{
María Yolanda Vellisca GonZÁlez*** \\ Universidad de Zaragoza, España \\ José IGNACIO LATORRE MARÍN *** \\ Hospital Obispo Polanco, Teruel, España \\ SANTOS OREJUDO HERNÁNDEZ ***** \\ Universidad de Zaragoza, España \\ José DAVID SAA SENDRA ****** \\ Hospital Obispo Polanco, Teruel, España \\ Miguel ÁNGEl SANTEd GERMÁN ******* \\ Universidad Nacional de Educación a Distancia,
}

Madrid, España

doi:10.11144/Javeriana.UPSY12-2.acda

Para citar este artículo: Vellisca, M. Y., Latorre, J. I., Orejudo, S., Saa, J. D. \& Santed, M. A. (2013). La amenorrea como criterio diagnóstico de la anorexia nerviosa. Universitas Psychologica, 12(2), 413-419.

* El presente artículo de investigación no ha recibido ningún tipo de financiación externa.

** Universidad de Zaragoza, Departamento de Psicología y Sociología, Teruel (España). C/Ciudad Escolar, s/n, 44003, Teruel. Tel: (34) 97861 81 01. E-mail: vellisca@unizar.es

**** Hospital Obispo Polanco, Departamento de Salud Mental, Teruel (España). C/ Jerónimo Soriano, s/n, 44002, Teruel. Tel: (34) 9786541 02. E-mail: latorre@cop.es

***** Universidad de Zaragoza, Departamento de Psicología y Sociología, Zaragoza (España). C/ San Juan Bosco, 7, 50009, Zaragoza. Tel: (34) 976 7610 01.E-mail: sorejudo@unizar.es

******** Hospital Obispo Polanco, Departamento de Salud Mental, Teruel (España). C/ Jerónimo Soriano, s/n, 44002, Teruel. Tel: (34) 9786541 02. E-mail: djsaa@salud.aragon.es

******* Universidad Nacional de Educación a Distancia, Departamento de Psicología de la Personalidad, Evaluación y Tratamiento Psicológico, Madrid (España). C/ Juan del Rosal, 10, 28040, Madrid. Tel: (34) 9023888 88. E-mail: msanted@psi. uned.es

\section{RES U MEN}

La amenorrea es un criterio para el diagnostico de la anorexia nerviosa (AN) según el DSM-IV-TR. Sin embargo, al comparar grupos de pacientes que cumplen todos los criterios de este manual para la AN con grupos de mujeres que los presentan todos salvo la amenorrea, algunos estudios no han encontrado diferencias significativas en la psicopatología típicamente asociadas con la AN. El propósito de nuestro estudio ha sido comparar variables demográficas, antropométricas, psicológicas y psicopatológicas, en ambos grupos. No se observaron diferencias estadísticamente significativas entre ambos grupos en las variables evaluadas, pero el grupo con amenorrea tenía un índice de masa corporal significativamente más bajo. Nuestros datos apoyan la hipótesis de que la amenorrea podría no ser un criterio diagnóstico útil para la $\mathrm{AN}$.

Palabras clave autores

Amenorrea, anorexia nerviosa, criterio diagnóstico, DSM-IV-TR.

Palabras clave descriptores

Psicología Clínica y de la Salud, Investigación Cuantitativa, DSM-IV-TR.

\footnotetext{
A B S T R A C T

Amenorrhea is a current criterion for the diagnosis of anorexia nervosa (AN) according to the DSM-IV-TR. Nevertheless, when comparing groups of patients who fulfill all the criteria of this manual for AN and groups of women who show them all but amenorrhea, some studies did not find significant differences in the psychopathology typically associated with AN. The purpose of our study was to compare both groups in demographic, anthropometric, psychological and psychopathological variables. There were no statistically significant differences between both groups in the analyzed variables, but the group with amenorrhea had a significantly lower body mass index. Our data support the hypothesis that amenorrhea could not be a useful criterion for AN.

Key words authors

Amenorrhea, Anorexia Nervosa, Diagnostic Criteria, DSM-IV-TR.

Key words plus

Clinical and Health Psychology, Quantitative Research, DSM-IV-TR.
} 


\section{Introducción}

La última edición revisada del Manual Diagnóstico y Estadístico de los Trastornos Mentales (DSM-IVTR) (American Psychiatric Association [APA], 2000), requiere para realizar el diagnostico de anorexia nerviosa (AN) la "ausencia de al menos tres ciclos menstruales consecutivos" (p. 660). La posibilidad de eliminar este criterio en la futura quinta edición del mencionado manual (DSM-V) ha sido considerada por varios autores (Abbate-Daga et al., 2010; Attia \& Roberto, 2009; Smith \& Wolfe, 2008). Finalmente, la APA (2012) ha propuesto la eliminación de dicho criterio de cara a la publicación del futuro DSM-V, ya que no resulta útil para el diagnóstico de los hombres, de las mujeres premenárquicas y posmenopáusicas, ni de aquellas que reciben terapia de reemplazo hormonal (ej., anticonceptivos orales). Además, muchas pacientes mantienen la menstruación a pesar de mostrar todos los demás criterios de la AN.

Cachelin y Maher (1998) compararon a un grupo de 40 mujeres diagnosticadas de AN con otro formado por 12 pacientes con síntomas de $\mathrm{AN}$ sin amenorrea (ANSA), que serían diagnosticadas según los criterios del DSM-IV-TR de trastorno de la conducta alimentaria (TCA) no especificado. El grupo de ANSA mostraba los mismos niveles de alteración de la alimentación y de la imagen corporal, de acuerdo con los resultados obtenidos en el Eating Disorder Inventory (EDI) de Garner, Olmstead y Polivy (1983) y en el Body Distortion Questionnaire de Fisher (1965), respectivamente. Del mismo modo, presentaban resultados equiparables en psicopatología, de acuerdo con las puntuaciones registradas en el Minnesota Multiphasic Personality Inventory de Hathaway y Mckinley (1940), y también resultaba equiparable la presencia de síntomas depresivos, según el Beck Depression Inventory (BDI) (Beck, Rush, Shaw \& Emery, 1979). La conclusión de estos autores es que la amenorrea no es un criterio útil para diferenciar entre casos "completos" e "incompletos" de AN.

En el estudio de Watson y Andersen (2003), las pacientes con ANSA $(N=49)$ mostraban niveles psicopatológicos similares a las que cumplían todos los criterios $(N=230)$, cuando eran comparadas sus puntuaciones en la versión abreviada de 26 ítem del Eating Attitudes Test (EAT) (Garner, Olmstead, Bohr \& Garfinkel, 1982) y en el BDI. Por este motivo concluyen que la amenorrea podría no ser un criterio diagnóstico útil.

Dalle-Grave, Calugi y Marchesini (2008) evaluaron las características clínicas de 57 pacientes con AN y las compararon con 16 casos de ANSA. No encontraron diferencias significativas entre ambos grupos al comparar los resultados en cuanto a alteraciones de la alimentación, medidas con el Eating Disorder Examination (EDE) de Fairburn y Cooper (1993); síntomas de depresión y ansiedad, medidos con el BDI y el State-Trait Anxiety Inventory de Spielberger, Gorsuch y Lushene (1970), respectivamente; ni tampoco en alteraciones de la personalidad, de acuerdo con el Temperament and Character Inventory de Cloninger, Svrakic, Przybeck y Wetzel (1994). La conclusión de los autores es que la amenorrea no tiene utilidad diagnóstica para la AN.

Roberto, Steinglass, Mayer, Attia y Walsh (2008) analizaron las puntuaciones obtenidas en el BDI, el Beck Anxiety Inventory (Beck, Epstein, Brown \& Steer, 1988) y el EDE en un grupo de 150 pacientes con $A N$ y el otro de 47 mujeres con ANSA. No encontraron diferencias significativas en estas variables, por lo que concluyeron que la amenorrea no es útil para diferenciar entre ambos grupos en cuanto a la gravedad de su trastorno.

La única diferencia que ha sido encontrada por alguno de estos autores (Cachelin \& Maher, 1998; Dalle-Grave et al., 2008) ha sido que las pacientes con amenorrea eran más jóvenes en el momento de ser admitidas a tratamiento, si bien otros investigadores no han podido replicar estas observaciones (Roberto et al., 2008; Watson \& Andersen, 2003).

El propósito del presente estudio fue evaluar la importancia de la amenorrea en el diagnóstico de la AN en una muestra clínica amplia. Para hacerlo, se comparó a un grupo de pacientes que cumplían todos los criterios del DSM-IV para la AN con otro grupo de ANSA. La evaluación incluyó variables demográficas, antropométricas, psicológicas y psicopatológicas. Se intentó replicar los resultados ob- 
servados en algunas de las variables estudiadas por los investigadores previos, y se incluyó la evaluación del nivel de autoestima de las pacientes, por haber sido ésta considerada una variable típicamente relacionada con la AN (ej., Karpowicz, Skärsäter \& Nevonen, 2009).

Este estudio supone el primer trabajo de envergadura que ha sido llevado a cabo con población clínica hispanoparlante. Los resultados apoyan la hipótesis de que no existen diferencias significativas entre ambos grupos en las variables relacionadas con la psicopatología típicamente asociada con la $\mathrm{AN}$, por lo que la amenorrea no resulta útil a la hora de hacer el diagnóstico de AN, y podría ser eliminada como criterio en el próximo DSM-V.

\section{Método}

\section{Participantes}

La muestra inicial estaba compuesta por las 1.041 personas que fueron atendidas durante un periodo de diez años en una unidad especializada en el tratamiento de TCA en España. Del total de las personas atendidas, se seleccionó a aquellas mujeres que cumplían todos los criterios diagnósticos del DSM-IV-TR para la AN y a las que los cumplían todos, salvo el de la amenorrea. Se consideró que no cumplían este criterio aquellas pacientes que habían tenido al menos una menstruación en los últimos tres meses. Para el objetivo de este estudio, se excluyó a las pacientes que estaban tomando anticonceptivos orales, a las embarazadas y a las que habían pasado la menopausia.

De esta forma, el primer grupo estaba compuesto por 176 mujeres diagnosticadas de $\mathrm{AN}$, de edades comprendidas entre los 18 y los 53 años $(\mathrm{M}=26.23, \mathrm{DE}=8.09)$. De las 176 pacientes con AN, 64 cumplía los criterios diagnósticos para el subgrupo de AN restrictiva y 112 para el subgrupo de AN compulsivo/purgativa. El grupo diagnosticado de AN restrictiva era algo más joven, con un rango de edades comprendidas entre los 18 y los 49 años $(M=24.62, D E=7.57)$, mientras que el diagnosticado de AN compulsivo/purgativa lo formaban mujeres con edades entre los 18 y los 53 años $(M=27.14, D E=8.27)$. Por último, el rango de edad de las 46 mujeres del grupo sin amenorrea se encontraba entre los 18 y los 51 años $(M=26.93$, $D E=8.04)$.

\section{Índice de Masa Corporal}

El Índice de Masa Corporal (IMC) fue calculado en todos los casos el primer día en el que las participantes eran admitidas a tratamiento en la Unidad. Para calcularlo se utilizó la fórmula peso/altura ${ }^{2}$, midiendo el peso en kilogramos y la altura en metros.

\section{Instrumentos}

Los criterios diagnósticos para la AN, incluida la amenorrea, fueron evaluados mediante la adaptación española de la entrevista EDE (Raich, Mora, Sánchez \& Torras, 2000); para evaluar la presencia de síntomas y preocupaciones característicos de la AN, se utilizó la versión española del EAT (Castro, Toro, Salamero \& Guimerá, 1991), que además proporciona un índice de la gravedad de dichos síntomas; la alteración de la imagen corporal propia de la AN fue medida con la adaptación española del Body Shape Questionnaire (BSQ) (Raich et al., 1996); la presencia de síntomas depresivos se evaluó mediante la versión española del BDI (Vázquez \& Sanz, 1991) y, para medir la autoestima, se empleó la adaptación española de la Self-Esteem Scale de Rosenberg (SES) (Baños \& Guillén, 2000).

\section{Procedimiento}

En la primera consulta, el psiquiatra de la unidad recoge, entre otros, los datos sociodemográficos y socioculturales de las pacientes y realiza un primer diagnóstico basándose en la entrevista clínica. Si considera que la derivación a la unidad de TCA es oportuna y que la paciente puede beneficiarse de una terapia psicológica, la remite a la consulta de la diplomada universitaria en enfermería y del psicólogo especialista en psicología clínica. La primera recoge, además de la información necesaria para llevar a cabo su propia labor, las medidas antropométricas (altura y peso) con las que se calcula el IMC. 
TABLA 1

Resultados del estadístico $t$ de Student para muestras independientes

\begin{tabular}{|c|c|c|c|c|c|c|c|c|c|}
\hline & \multicolumn{6}{|c|}{ Anorexia Nerviosa } & \multirow{3}{*}{$t$} & \multirow{3}{*}{$d f$} & \multirow{3}{*}{$p$} \\
\hline & \multicolumn{3}{|c|}{ Anorexia Nerviosa } & \multicolumn{3}{|c|}{ Sin Amenorrea } & & & \\
\hline & $\mathrm{N}$ & M & $D E$ & $\mathrm{~N}$ & $M$ & $D E$ & & & \\
\hline Edad (años) & 176 & 26.22 & 8.09 & 46 & 26.93 & 8.04 & -0.529 & 220 & 0.598 \\
\hline $\operatorname{IMC}\left(\mathrm{kg} / \mathrm{m}^{2}\right)$ & 176 & 14.79 & 1.79 & 46 & 16.8 & 2.02 & -6.594 & 220 & 0.000 \\
\hline EAT & 136 & 49.47 & 26.25 & 42 & 45.35 & 24.21 & 1.586 & 176 & 0.097 \\
\hline BSQ & 166 & 118.93 & 38.35 & 43 & 106.78 & 48.44 & 1.748 & 207 & 0.082 \\
\hline BDI & 170 & 24.67 & 12.53 & 42 & 21.28 & 10.92 & 1.61 & 210 & 0.11 \\
\hline SES & 168 & 13.1 & 5.43 & 40 & 13.61 & 5.71 & -0.528 & 206 & 0.598 \\
\hline
\end{tabular}

Nota IMC = Índice de Masa Corporal; EAT = Eating Attitudes Test (Castro et al., 1991); BSQ = Body Shape Questionnaire (Raich et al., 1996); BDI = Beck Depression Inventory (Vázquez \& Sanz, 1991); SES = Self-Esteem Scale (Baños \& Guillén, 2000).

Fuente: elaboración propia.

Por su parte, el psicólogo clínico, basándose en la entrevista clínica y en la EDE, recaba de manera sistemática el resto de los datos y realiza su propio diagnóstico. Para evitar un excesivo cansancio a la hora de cumplimentar los cuestionarios puede ser necesario dejar algunos para una segunda sesión. La inasistencia de varias de las pacientes a la segunda sesión con el psicólogo clínico explica que en algunos casos no se cuente con la información de todos los test.

\section{Análisis estadísticos}

Se utilizo el estadístico $t$ de Student para muestras independientes a la hora de comparar a las pacientes con y sin amenorrea. Se consideró significativo un valor de $p$ menor de 0.05 .

\section{Resultados}

Las características de las pacientes de cada grupo diagnóstico se muestran en la Tabla 1 . Se registró la edad, la altura y el peso de todas ellas. El número de test válidos para cada caso también se recoge en la misma tabla.

Tal y como se anticipó en las hipótesis, no se observaron diferencias estadísticamente significativas al comparar el grupo que cumplía todos los criterios diagnósticos de la AN con el que no cumplía el criterio de amenorrea. La única excepción fue el IMC, que resultó significativamente menor $(t=-6.594 ; g l=220 ; p<0.001)$ en el grupo con amenorrea. No se observó ninguna otra diferencia significativa en cuanto a edad $(t=-0.529 ; g l=220$; $p=0.598)$, gravedad de los síntomas medida con el EAT $(t=1.586 ; g l=176 ; p=0.097)$, insatisfacción corporal de acuerdo con el BSQ $(t=1.748$; $g l=207 ; p=0.082)$, sintomatología depresiva según el BDI $(t=1.61 ; g l=210 ; p=0.11)$, ni tampoco en el nivel de autoestima medido con el SES $(t=-0.528 ; g l=206 ; p=0.598)$.

\section{Discusión}

De acuerdo con lo observado en trabajos previos (Abbate-Daga et al., 2010; Abraham, Pettigrew, Boyd, Russell \& Taylor, 2005; Attia \& Roberto, 2009; Smith \& Wolfe, 2008), en nuestro estudio apenas se observaron diferencias en las variables evaluadas entre las pacientes con AN y las que presentaban ANSA. Ambos grupos resultaban estadísticamente equiparables en cuanto a su edad, tal y como otros autores han observado (Roberto et al., 2008; Watson \& Andersen, 2003), si bien otros estudios (Cachelin \& Maher, 1998; DalleGrave et al., 2008) han advertido que sus muestras de mujeres con amenorrea eran más jóvenes en el momento de ser admitidas a tratamiento. Cabe resaltar que estos últimos estudios, en los que sí que se observaban diferencias en función de la edad, utilizaron en sus comparaciones grupos de pacientes sin amenorrea de un tamaño menor $(N=12$ y 
$N=16$, respectivamente), por lo que su capacidad para extrapolar sus observaciones a otras muestras se reduce.

Los dos grupos observados en el presente estudio puntuaban de manera equivalente en el EAT, revelando con ello la presencia de síntomas de gravedad similar. Watson y Andersen (2003) encontraron la misma ausencia de diferencias, utilizando la versión abreviada de este test. Por lo que respecta a la distorsión de la imagen corporal, nuestros datos son similares a los aportados por Cachelin y Maher (1998), que no encontraron diferencias significativas al comparar las puntuaciones obtenidas en el Body Distortion Questionnaire por grupos con y sin amenorrea. Con respecto a los síntomas depresivos, en la presente investigación revela la ausencia de diferencias significativas entre los grupos, en consonancia con lo observado en estudios previos (Cachelin \& Maher, 1998; DalleGrave et al., 2008; Roberto et al., 2008; Watson \& Andersen, 2003). Finalmente, por lo que respecta a los resultados del SES, los análisis tampoco revelaron la existencia de diferencias significativas en los niveles de autoestima entre ambos grupos. No se encontró en la revisión bibliográfica ningún estudio previo que hubiese analizado esta variable, no obstante, la ausencia de diferencias está en consonancia con las observaciones de los estudios previamente comentados.

Estas observaciones sugieren que las características psicopatológicas de ambos grupos son similares. Las pacientes que, siguiendo los criterios diagnósticos del DSM-IV-TR, son diagnosticadas de TCA no especificado por no cumplir el criterio de amenorrea presentan gravedad sintomática, distorsión de su imagen corporal, síntomas depresivos y un nivel de autoestima que resultan estadísticamente similares a los observados en las pacientes diagnosticadas de AN.

La única diferencia que ha sido constatada de forma consistente es la relativa al IMC. En consonancia con los estudios previos (Abraham et al., 2005; Cachelin \& Maher, 1998; Dalle-Grave et al., 2008; Roberto et al., 2008; Watson \& Andersen, 2003), el grupo diagnosticado de AN presentaba un IMC significativamente más bajo que el de
ANSA en el momento de admisión. Todos estos datos sugieren que la amenorrea está relacionada con la pérdida de IMC y no con la psicopatología o las variables de personalidad características de la AN. Por todo lo anterior, se apoya la propuesta de que se retire la amenorrea de los criterios diagnósticos de la AN en el próximo DSM-V, tal y como han sugerido previamente otros autores (Dalle-Grave et al., 2008; Fairburn et al., 2007; Mitchell, Cook-Myers \& Wonderlich, 2005; Watson \& Andersen, 2003). Los datos respaldan la hipótesis de que la amenorrea, siendo importante en la evaluación del estado de salud de las pacientes, podría no ser un criterio diagnóstico útil.

Como limitaciones de este estudio, se ha de considerar en primer lugar el tamaño de nuestra muestra, especialmente la del grupo de ANSA. Este es un problema común en la investigación de la AN. Una muestra mayor hubiera incrementado la potencia estadística de esta investigación, por lo que resultará deseable la replicación de las observaciones en muestras más amplias. Otra de las limitaciones reseñables es la pérdida de los datos de algunos test debida a la inasistencia de varias pacientes a su segunda sesión. No obstante, pese a que la pérdida de datos resta poder estadístico al estudio, se considera que las muestras utilizadas, correspondientes a todas las pacientes atendidas en una unidad especializada en el tratamiento de TCA durante diez años, siguen siendo suficientemente significativas. A pesar de estas limitaciones, los hallazgos resultan consistentes con las observaciones previas de otros estudios.

\section{Referencias}

Abbate-Daga, G., Notaro, G., Quaranta, M., Urani, C., Barbieri, A. \& Fassino, S. (2010). Anoressia nervosa: Review sul criterio diagnostico amenorrea [Anorexia nervosa: A diagnostic criterion amenorrhea review]. Minerva Psichiatrica, 51(2), 127-133.

Abraham, S., Pettigrew, B., Boyd, C., Russell, J. \& Taylor, A. (2005). Usefulness of amenorrhea in the diagnoses of eating disorder patients. Journal of 
Psychosomatic Obstetrics $\mathcal{G}$ Gynecology, 26(3), 211215. doi: $10.1080 / 01674820500064997$

American Psychiatric Association. (2000). Diagnostic and statistical manual of mental disorders (text revision). Washington, DC: Autor.

American Psychiatric Association. (2012). DSM-5 development: K03 Anorexia Nervosa. Recuperado el 15 de julio, 2012, de http://www.dsm5.org/ProposedRevision/Pages/proposedrevision.aspx?rid=24\#

Attia, E. \& Roberto, C. A. (2009). Should amenorrhea be a diagnostic criterion for anorexia nervosa? International Journal of Eating Disorders, 42(7), 581-589. doi: 10.1002/eat.20720

Baños, R. M. \& Guillén, V. (2000). Psychometric characteristics in normal and social phobic samples for a Spanish version of the Rosenberg Self-Esteem Scale. Psychological Reports, 87(1), 269-274.

Beck, A. T., Epstein, N., Brown, G. \& Steer, R. A. (1988). An inventory for measuring clinical anxiety: Psychometric properties. Journal of Consulting and Clinical Psychology, 56(6), 893-897.

Beck, A. T., Rush, A. J., Shaw, B. F. \& Emery, G. (1979). Cognitive therapy of depression. New York: Guilford Press.

Cachelin, F. M. \& Maher, B. A. (1998). Is amenorrhea a critical criterion for anorexia nervosa? Journal of Psychosomatic Research, 44(3-4), 435-440. doi: 10.1016/S0022-3999(97)00268-7

Castro, J., Toro, J., Salamero, M. \& Guimerá, E. (1991). The Eating Attitudes Test: Validation of the Spanish version. Psychological Assessment, 7(2), 175-190.

Cloninger, C. R., Svrakic, D. M., Przybeck, T. R. \& Wetzel, R. D. (1994). The Temperament and Character Inventory (TCI): A guide to its development and use. St. Louis, MO: Center for Psychobiology of Personality.

Dalle-Grave, R., Calugi, S. \& Marchesini, G. (2008). Is amenorrhea a clinically useful criterion for the diagnosis of anorexia nervosa? Behaviour Research and Therapy, 46(12), 1290-1294. doi: 10.1016/j. brat.2008.08.007

Fairburn, C. G. \& Cooper, Z. (1993). The Eating Disorder Examination. In C. G. Fairburn \& G. T. Wilson (Eds.), Binge eating: Nature, assessment and treatment (pp. 317-360). New York: Guilford Press.
Fairburn, C. G., Cooper, Z., Bohn, K., O’Connor, M. E., Doll, H. A. \& Palmer, R. L. (2007). The severity and status of eating disorder NOS: implications for DSM-V. Behaviour Research and Therapy, 45(8), 1705-1715. doi: 10.1016/j.brat.2007.01.010

Fisher, S. (1965). The body image as a source of selective cognitive sets. Journal of Personality, 33(4), 536-552. doi: 10.1111/j.1467-6494.1965.tb01401.x

Garner, D. M., Olmstead, M. P., Bohr, Y. \& Garfinkel, P. E. (1982). The Eating Attitudes Test: Psychometric features and clinical correlates. Psychological Medicine, 12(4), 871-878. doi: 10.1017/ S0033291700049163

Garner, D. M., Olmstead, M. P. \& Polivy, J. (1983). Development and validation of a Multidimensional Eating Disorder Inventory for Anorexia Nervosa and Bulimia. International Journal of Eating Disorders, 2(2), 15-34. doi: 10.1002/1098-108X(198321)2:2<15::AID EAT2260020203>3.0.CO;2-6

Hathaway, S. R. \& Mckinley, J. C. (1940). A Multiphasic Personality Schedule (Minnesota): I. Construction of the schedule. The Journal of Psychology, 10(2), 249-254.

Karpowicz, E., Skärsäter, I. \& Nevonen, L. (2009). Selfesteem in patients treated for anorexia nervosa. International Journal of Mental Health Nursing, 18(5), 318-325. doi: 10.1111/j.1447-0349.2009.00621.x

Mitchell, J. E., Cook-Myers, T. \& Wonderlich, S. A. (2005). Diagnostic criteria for anorexia nervosa: Looking ahead to DSM-V. International Journal of Eating Disorders, 37(1), 95-97. doi: 10.1002/ eat.20125

Raich, R. M., Mora, M., Sánchez, D. \& Torras, J. (2000). Adaptación y calidad psicométrica de la entrevista EDE (Eating Disorder Examination) para la evaluación de los trastornos alimentarios en población universitaria. Revista de la Asociación Española para el Estudio de los Trastornos de la Conducta Alimentaria, 1(6), 3-5.

Raich, R. M., Mora, M., Soler, A., Ávila, C., Clos, I. \& Zapater, L. (1996). Adaptación de un instrumento de evaluación de la insatisfacción corporal. Clínica y Salud, 1(7), 51-66.

Roberto, C. A., Steinglass, J., Mayer, L. E., Attia, E. \& Walsh, B. T. (2008). The clinical significance of 
amenorrhea as a diagnostic criterion for anorexia nervosa. International Journal of Eating Disorders, 41(6), 559-563. doi: 10.1002/eat.20534

Smith, A. T. \& Wolfe, B. E. (2008). Amenorrhea as a diagnostic criterion for anorexia nervosa: A review of the evidence and implications for practice. Journal of the American Psychiatric Nurses Association, 14(3), 209-215. doi: 10.1177/1078390308320288

Spielberger, C. D., Gorsuch, R. L. \& Lushene, R. E. (1970). Manual for the State-Trait Anxiety Inventory (self evaluation questionnaire). Palo Alto: Consulting Psychologists Press.
Vázquez, C. \& Sanz, J. (1991, septiembre). Fiabilidad y validez factorial de la versión española del Inventario de Depresión de Beck. Comunicación presentada en el III Congreso de Evaluación Psicológica, Barcelona, España.

Watson, T. L. \& Andersen A. E. (2003). A critical examination of the amenorrhea and weight criteria for diagnosing anorexia nervosa. Acta Psychiatrica Scandinavica, 108(3), 175-182. doi: 10.1034/j.16000447.2003.00201.x 
